\title{
Current Conveyor Equation-Defined Macromodels for Wideband RF Circuit Design
}

\author{
Mike Brinson, and Vadim Kuznetsov
}

\begin{abstract}
A high percentage of analogue integrated circuit designs use voltage domain signal processing techniques. Given the fact that integrated circuit current conveyors are high bandwidth current processing devices, often with superior RF performance compared to comparable voltage domain devices, it is surprising that the number of current mode integrated circuits available, as standard of-the-shelf industrial items, is so small. This paper introduces equation-defined device and Verilog-A synthesis approaches to the macromodelling of current conveyor integrated circuits. To illustrate the proposed modelling techniques the properties of a number of modular behavioural level current conveyor macromodel cells are described and their performance compared. The material presented is intended for analogue device modellers and circuit designers who wish to simulate large signal current domain integrated circuit designs. It also demonstrates how synthesized Verilog-A modules can be derived from equation-defined device and conventional subcircuits to form functional, computationally efficient current conveyor macromodels. To illustrate the application of behavioural current conveyor macromodels the design of a six cell $\mathrm{CCII}+$ instrumentation amplifier is introduced and its performance discussed.
\end{abstract}

Index Terms-Qucs, current conveyors, compact semiconductor device modelling, equation-defined devices, macromodels, Verilog-A module synthesis.

\section{INTRODUCTION}

B EHAVIOURAL modelling of integrated circuits with macromodels has evolved into an established modelling technique [1], particularly after Boyle et al. [2] published a computationally efficient operational amplifier macromodel that included a mixture of first and second order device charcteristics. A macromodel in this context implies a collection of linear and non-linear simulation component models, combined as a subcircuit, where the simulated electrical signals at the subcircuit input-output pins appear to be similar to those generated by a physical device. Adding Qucs equation-defined device (EDD) technology $[3,4]$ to behavioural macromodelling introduces a powerful algebraic capability for specifying the non-linear properties of semiconductor devices and integrated circuits. This approach has become more firmly entrenched with the adoption of the Verilog-A hardware description language for compact semiconductor device modelling [5][16]. Although Verilog-A device modelling is well established for compact semiconductor device model construction, its use for conventional circuit macromodelling is often assumed or implied by the nature of the hardware description language. This paper proposes a structured route for behavioural

M. Brinson is with the Centre for Communications Technology, London Metropolitan University, UK (e-mail: mbrin72043@yahoo.co.uk)

V. Kuznetsov is with the Department of Electronic Engineering, Bauman Moscow State Technical University, Kaluga branch, Russia (e-mail: ra3xdh@gmail.com) compact macromodelling from original specification through preliminary model design, with equation-defined defined device technology, to a final optimized Verilog-A module, via model synthesis. To demonstrate the proposed macromodelling procedure the properties and relationships between current conveyor macromodels, at different levels of circuit abstraction and signal levels, are described. A number of Qucs [6] and Qucs-S [17] simulation test-bench circuits, plus typical simulation data are also included, and their performance compared.

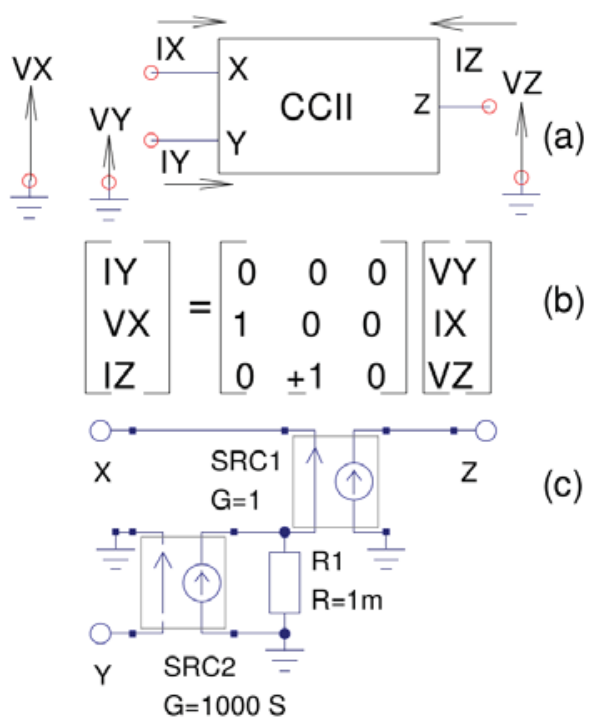

Fig. 1. Specification for a second generation current conveyor macromodel: (a) Qucs schematic symbol, (b) port current/voltage relationships in matrix form, (c) a first level ideal macromodel.

\section{Current Conveyor Principles}

The first generation current conveyor (CCI) was introduced by Sedra and Smith in 1968 [7] as a three port circuit building block for analogue signal processing. In 1970 the same authors [8] reported an improved second generation device (CCII) which overcame a number of limitations inherent in the original device specification. Today the CCII current conveyor is an RF mode device which finds application in wideband amplification, filtering and other signal processing functions $[9,10]$. An additional source of reference for current conveyor principles and RF applications can be found in the IEE publication edited by Toumazou, Lidgy and Haigh [15]. A schematic symbol for a CCII is shown in Fig. 1 (a). The ideal current/voltage relationships at the device ports are $I Y=0$ (high input impedance at $Y$ terminal $\rightarrow \infty$ ), $V X=V Y$ (low input impedance at $X$ terminal $\rightarrow 0$ ) and $I Z= \pm I X$ (high output impedance at $Z$ terminal $\rightarrow \infty$ ). These relationships can be expressed in the matrix form given in Fig. 1 (b), where 
the \pm sign in equation $I Z= \pm I X$ signifies the direction of $I Z$ current flow in comparison to current $I X$ : called $C C I I+$ when $I Z=I X$ and $C C I I-$ when $I Z=-I X$. Fig. 1 (c) introduces a level one macromodel of a CCII device, where the electrical properties of the input and output ports are set by linear voltage controlled current source SRC2, which functions as a voltage follower, and by current controlled current source SRC1, which acts as a current follower. The level one CCII macromodel represents an ideal device that has an infinite signal frequency bandwidth, see the current mode amplifier shown in Fig. 2.
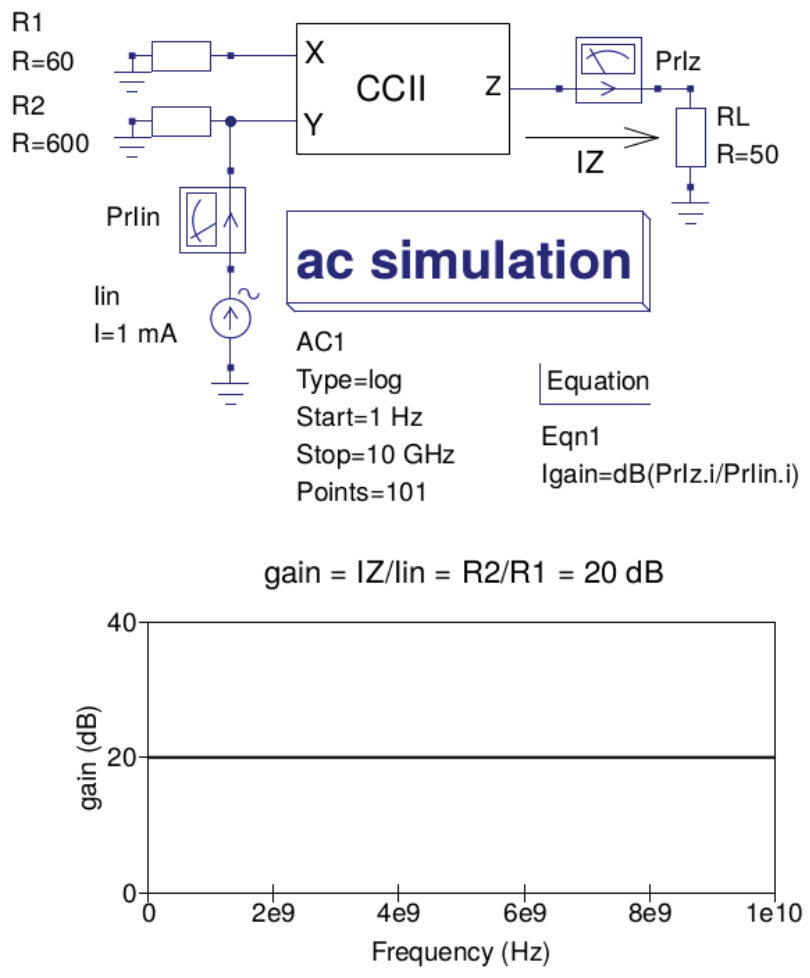

Fig. 2. A current conveyor current amplifier: gain $=I Z /$ Iin $=R 2 / R 1$.

\section{QuCs EDD MACROMOdELS AT DiFFERENT LEVELS of Circuit ABStraction}

For integrated circuit macromodels to be useful design aids they must be stable and function without convergence problems in all circuit simulation domains, including the steady state AC large signal, Harmonic Balance and transient shooting method domains. They should also be defined at different levels of circuit abstraction from ideal to non-linear, allowing choice of macromodel to meet the requirements of a given circuit simulation. A significant improvement in macromodel simulation run-time performance can sometimes result with circuit simulators which include Verilog-A synthesisers/compilers that generate $\mathrm{C}++$ code level models built with Verilog-A modules [11,12] embedded in subcircuits. In this paper five macromodel circuit abstraction levels are proposed, four at circuit schematic level, ideal to non-linear, and one at Verilog-A module level, see Table I. Examples of the large signal performance of the non-linear level current conveyor macromodel are reported in sections IV and $\mathrm{V}$.
These present transient and Harmonic Balance data obtained from Ngspice [18] large signal FFT simulation and Xyce [19] Harmonic balance simulation.

TABLE I

MACROMODEL LEVELS OF CIRCUIT ABSTRACTION.

\begin{tabular}{|c|c|c|}
\hline Level & Model Abstraction & Properties \\
\hline 1 & Ideal & $\begin{array}{c}\text { Fundamental current transfer } \\
\text { characteristic }\end{array}$ \\
\hline 2 & Linear DC & $\begin{array}{l}\text { Level } 1 \text { plus input and output } \\
\text { resistance and gain parameters }\end{array}$ \\
\hline 3 & Small signal AC & Level 2 plus internal capacitance \\
\hline 4 & $\begin{array}{c}\text { Large signal } \\
\text { non-linear }\end{array}$ & $\begin{array}{c}\text { Level } 3 \text { plus nonlinear device } \\
\text { parameters, including offset } \\
\text { current and saturation effects }\end{array}$ \\
\hline 5 & Verilog-A & $\begin{array}{l}\text { Verilog-A module representation } \\
\text { of macromodels levels } 1 \text { to } 4\end{array}$ \\
\hline
\end{tabular}

\section{A. Level 2 Current Conveyor Macromodel}

The simple level one macromodel shown in Fig. 1 (c) assumes that the properties of a current conveyor are ideal with controlled sources that have matched unity gain transfer functions, the $X$ port input resistance is zero and that the $Y$ and $Z$ ports have infinite input and output resistance, respectively. In reality a real current conveyor has properties which are far from ideal. Fig. 3 introduces the macromodel for the next level of circuit extraction. This adds port resistors $R X, R Y$ and $R Z$ and the gain parameters $P 1$ and $P 2$. The latter two determine how close the voltage and current follower transfer functions are to unity. Component $R X$ and the current $I 2$ flowing through $E D D-D 2$ sense current $I X=(V(n X)-V(n 1)) / R X$, from which conveyor current $I Z$ is calculated as $I Z=P 2 * V 1 /(1 e-6+R X)$, where $V 1$ is voltage drop across $E D D-D 1$ branch one. The graph of gain against frequency illustrated in Fig. 3 is for data obtained with the simulation test bench shown in Fig. 2 and $R 1=10 \Omega$ to compensate for port $I X$ internal resistance of $50 \Omega$.

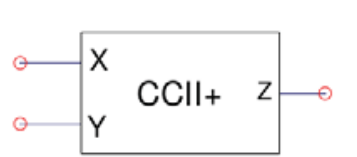

CCII1

$\mathrm{P} 1=1.12$

$\mathrm{P} 2=0.927$

$\mathrm{RX}=50$

$\mathrm{RY}=10 \mathrm{e} 6$
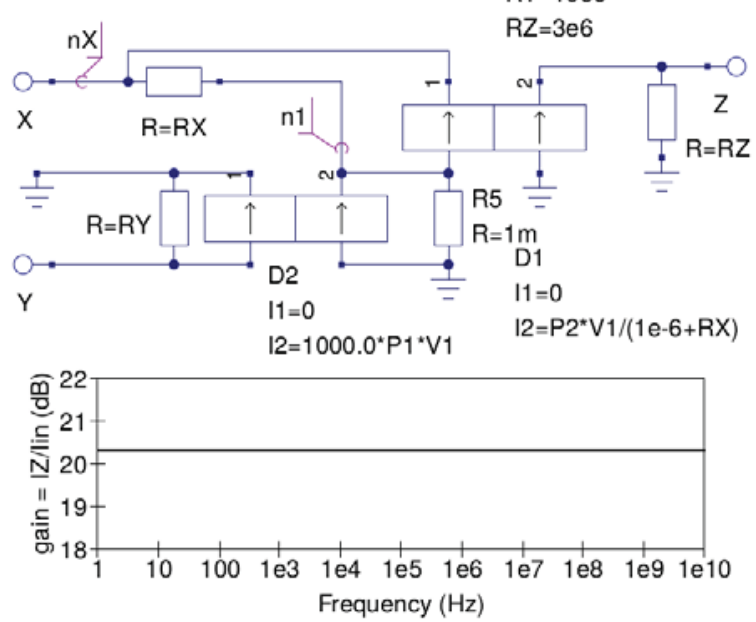

Fig. 3. Level 2 current conveyor macromodel: $R X=50 \Omega, R Y=10 \mathrm{M} \Omega$, $R Z=3 \mathrm{M} \Omega, P 1=1.12$ and $P 2=0.927$. 
The level one and level two CCII macromodels have an infinite frequency bandwidth. This, of course, is not true for a real current conveyor integrated circuit. In reality all three device ports have a finite capacitance connected to ground which causes the current gain frequency response to decrease at high frequencies. A level three CCII+ macromodel is shown in Fig. 4. With the port capacitors set to $4.5 \mathrm{pF}$, small signal AC simulation indicates that the current amplifier has two poles between $50 \mathrm{MHz}$ and $800 \mathrm{MHz}$ resulting in $40 \mathrm{~dB}$ per decade roll-off in the gain response at frequencies above $1 \mathrm{GHz}$. Frequency domain analysis of the level three CCII+ macromodel yields the following transfer function equations

$$
\begin{gathered}
V(n 1)=\frac{R 2 \cdot R Y \cdot P 1 \cdot \text { Iin }}{(R 2+R Y) \cdot\left[1+j \cdot \frac{\omega}{\omega_{y p}}\right]} \\
I X=\frac{\left[1+j \cdot \frac{\omega}{\omega x z}\right] \cdot V(n 1)}{(R 1+R X) \cdot\left[1+j \cdot \frac{\omega}{\omega x p}\right]} \\
I Z=\frac{P 2 \cdot I X}{\left[1+j \frac{\omega}{\omega z p}\right]}
\end{gathered}
$$

where $V(n 1)$ is the voltage at node $n 1$, see Fig. 4 , and

$$
\begin{gathered}
\omega y p=\frac{(R 2+R y)}{C Y \cdot R 2 \cdot R Y} \\
\omega x z=\frac{1}{C X \cdot R 1} \\
\omega x p=\frac{(R 1+R X)}{C X \cdot R 1 \cdot R X} \\
\omega z p=\frac{(R Z+R L)}{C Z \cdot R Z \cdot R L}
\end{gathered}
$$

With $R 1=10 \Omega$ and $R X=50 \Omega, \omega x z \approx \omega x p$ indicating that pole-zero cancellation takes place in the expression for $I X$, yielding

$$
\frac{I X}{I i n}=\frac{P 1 \cdot P 2 \cdot R 2 \cdot R Y}{(R 1+R X) \cdot(R 2+R Y) \cdot\left[1+j \cdot \frac{\omega}{\omega y p}\right] \cdot\left[1+j \cdot \frac{\omega}{\omega z p}\right]}
$$

where $f y p=\omega y p /(2 \cdot \pi)=707.36 \mathrm{MHz}$ and $f z p=\omega z p /(2 \cdot \pi)$ $=58.94 \mathrm{MHz}$ for the CCII+ parameter values listed in the caption to Fig. 4 and $R L=50 \Omega$.

Qucs-0.0.19-S/Ngspice .PZ pole-zero analysis confirms the three pole and the single zero frequencies, see Table II.
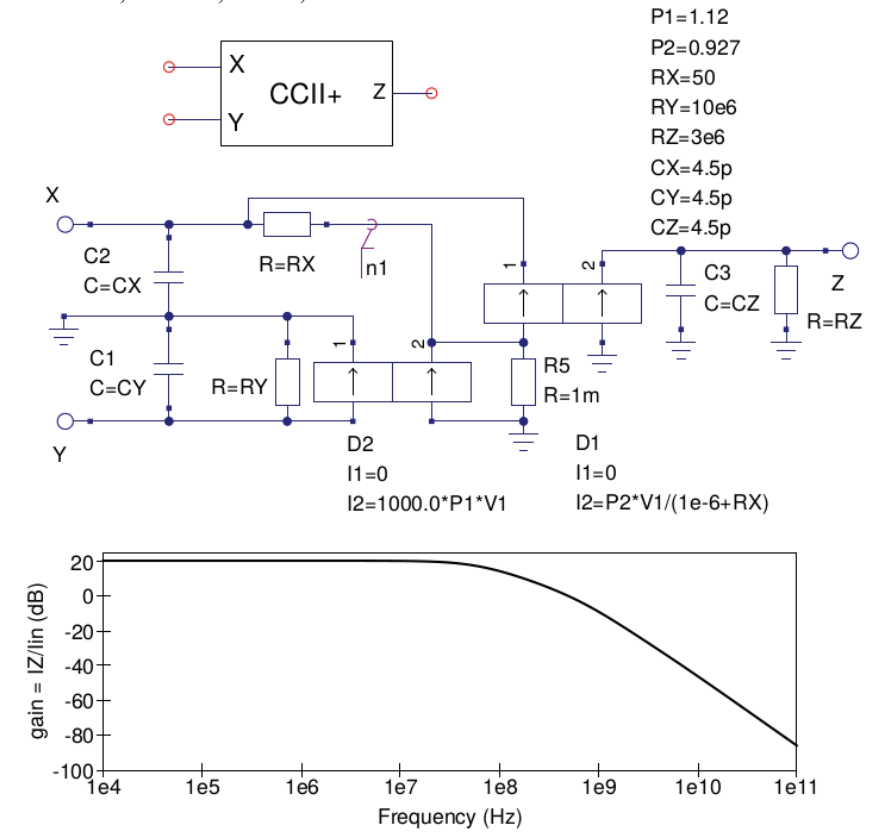

Fig. 4. Level 3 current conveyor macromodel: $R X=50 \Omega, R Y=10 \mathrm{M} \Omega$, $R Z=3 \mathrm{M} \Omega, P 1=1.12$ and $P 2=0.927, C X=C Y=C Z=4.5 \mathrm{pF}$.

TABLE II

LEVEL THREE CCII MACROMODEL POLE-ZERO FREQUENCIES.

\begin{tabular}{|c|c|c|}
\hline Pole number & $\omega_{p}$ (radians) & $f_{p}(\mathbf{H z})$ \\
\hline \hline 1 & $-2.67 \mathrm{e} 10$ & $-4.25 \mathrm{e} 9$ \\
2 & $-4.44 \mathrm{e} 9$ & $-707.66 \mathrm{e} 6$ \\
3 & $-3.70 \mathrm{e} 8$ & $-58.89 \mathrm{e} 6$ \\
\hline Zero number & $\omega_{z}$ (radians) & $f_{z}(\mathbf{H z})$ \\
\hline 1 & $-2.22 \mathrm{e} 10$ & $-3.53 \mathrm{e} 9$ \\
\hline
\end{tabular}

\section{Level 4 Current Conveyor Macromodel}

Level four current conveyor macromodels are applicable where non-linear circuit properties have a critical effect on simulation accuracy. One extension to the level three CCII+ macromodel, which is necessary to simulate large signal performance correctly, is a circuit extension that handles CCII+ operation at signal levels approaching, and above or below, DC supply voltages. An example of a level four CCII+ macromodel is shown in Fig. 5 where the voltage follower output voltage $V(n 1)$ is modelled by a tanh limiter function given by

$V(n 1)=P 1 \cdot V(Y) \cdot(1.0-\tanh (\operatorname{abs}(V(Y) /($ Scale $\cdot V M A X))$

where $-V M A X$ and $+V M A X$ represent the CCII+ negative and positive DC supply voltages, and Scale is a scaling factor which determines the degree of limiting at voltages greater than $+V M A X$ or less than $-V M A X$.

A suitable value for parameter Scale can be found by simulating the performance of a unity gain CCII+ current amplifier with $V M A X$ kept constant and Scale scanned over finite range, see Fig. 6 . When parameter Scale is set to a large value, for example $\geq 10$, the level four CCII+ macromodel reverts to the level three macromodel. With Scale $=2.7$ the CCII+ current amplifier peak output current $I Z$ and peak output voltage $V(Z)$ are effectively clamped to $20 \mathrm{~mA}$ and $10 \mathrm{~V}$ respectively, see Fig. 7. 


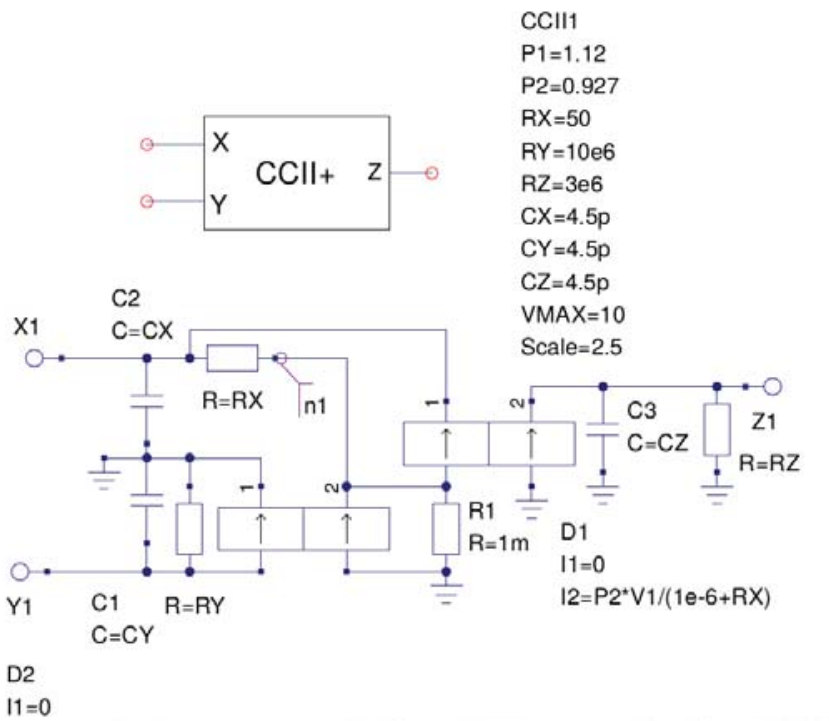

$12=1000.0^{*} \mathrm{P} 1{ }^{*} \mathrm{~V} 1{ }^{*}(1.0-(\sinh (\operatorname{abs}(\mathrm{V} 1) /($ Scale*VMAX $)) / \cosh (\operatorname{abs}(\mathrm{V} 1) /($ Scale*VMAX $))))$

Fig. 5. Level 4 current conveyor macromodel: DC power supply voltage $V M A X=10 \mathrm{~V}$ and scale factor Scale $=2.5$.
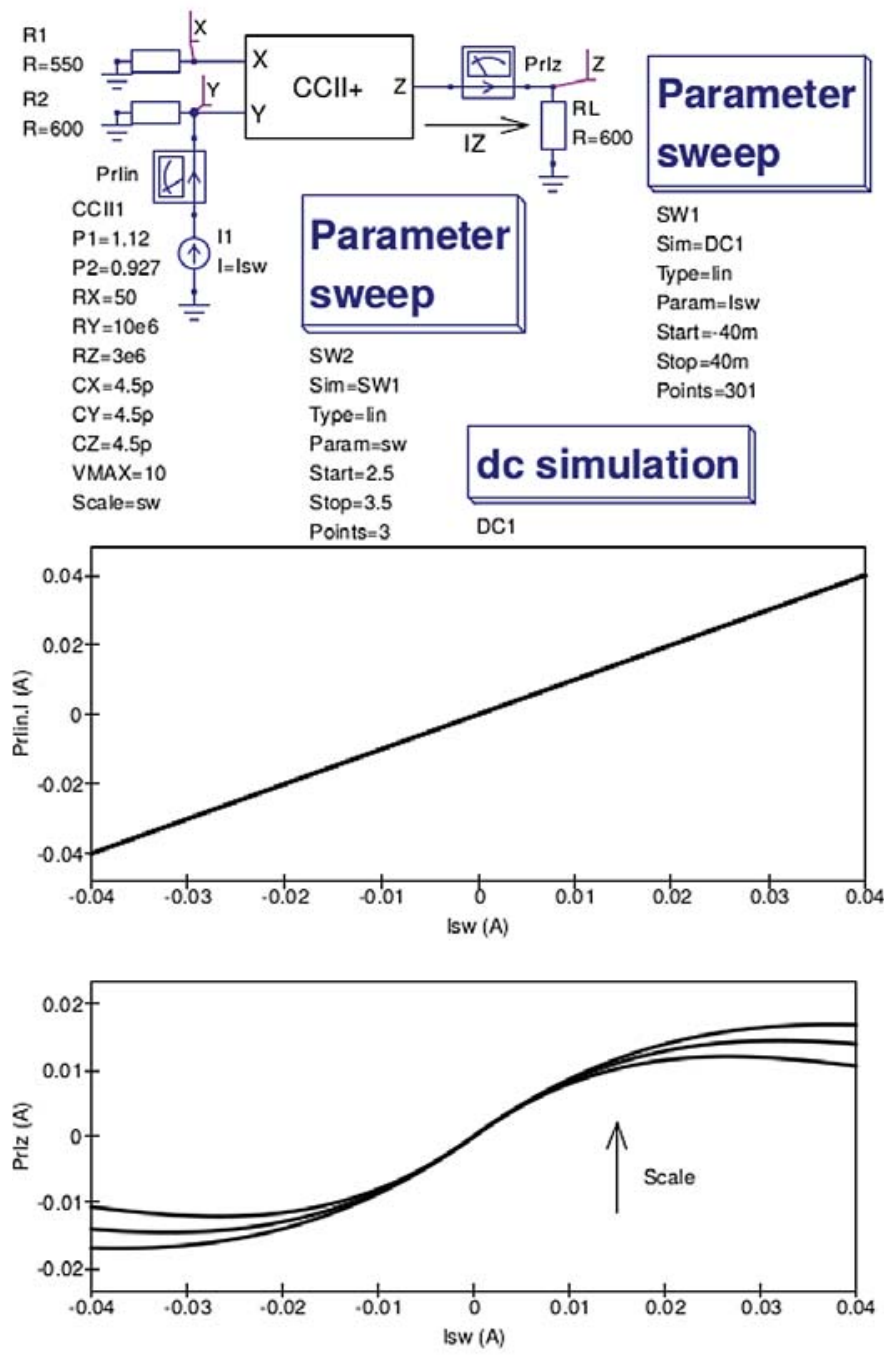

Fig. 6. Level 5 unity gain current amplifier: DC power supply voltage $V M A X=10 \mathrm{~V}$ and $2.5>=$ Scale $<=3.5$.
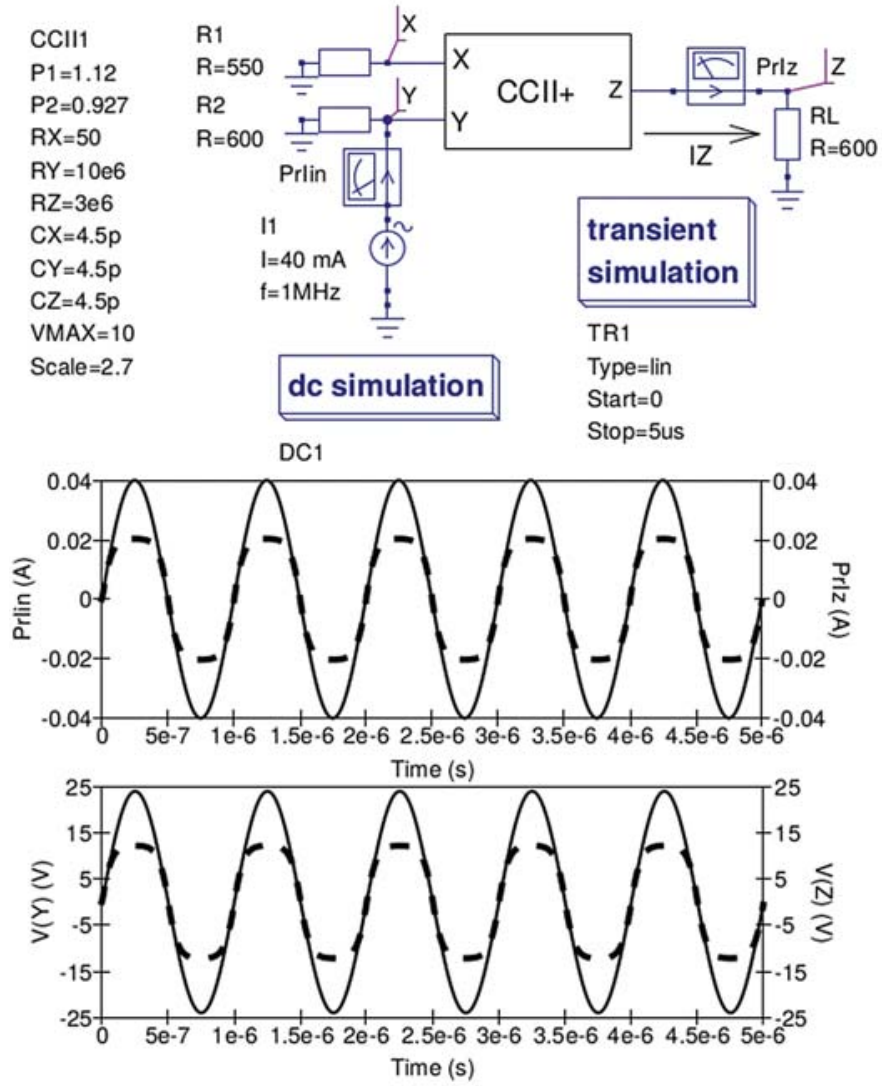

Fig. 7. Transient simulation of a unity gain CCII+ current amplifier: test circuit and plotted current and voltage data; left side scale solid line and right side scale dotted line.

\section{Level 5 Current Conveyor Macromodel}

In many design projects conventional macromodels are more than adequate for simulating circuit performance. However, as the size of a macromodel circuit increases, or if its circuit is very non-linear, simulation may require significant amounts of computing time. Replacement of complex circuit level macromodels by low level $\mathrm{C}$ or $\mathrm{C}++$ code models synthesized from the Verilog-A hardware description language can sometimes reduce circuit simulation time, significantly. However, this statement is not meant to imply that macromodel levels one to four are redundant but rather that they are simply stages in a structured process for generating computationally efficient Verilog-A macromodels. Recent developments in Qucs compact device modelling capabilities have shown that Verlog-A modules can be synthesized automatically from circuit schematics $[13,14]$, yielding a direct link between the level one to level four CCII macromodels and compiled $\mathrm{C}++$ macromodels. The Qucs Verilog-A model synthesiser allows macromodels or compact device models to be constructed from the Qucs and SPICE components listed in Table III. These are a mixture of linear passive components $\mathrm{R}, \mathrm{C}$ and L, controlled sources VCCS, CCCS, VCVS and CCVS, nonlinear components EDD and $\mathrm{B}$, an algebraic equation block EQN and subroutine features PIN, SUB and SPARAM. The synthesized Verilog-A module code for a level five CCII+ macromodel is listed in Fig. 8. The Qucs Verilog-A synthesizer automatically adds white noise current to each macromodel resistor. However, to simulate CCII+ noise correctly additional noise contributions must be added to the macromodel voltage 
and current followers. This can be done using Qucs INOISE generators with their shot and flicker noise outputs set at appropriate values.

TABLE III

BUILT-IN QUCS/SPICE COMPONENTS FOR CONSTRUCTING VERILOG-A SYNTHESIZED MACROMODELS AND DEVICE MODELS.

\begin{tabular}{|c|c|c|c|}
\hline $\begin{array}{c}\text { Component } \\
\text { code }\end{array}$ & Type & Qucs & SPICE \\
\hline \hline $\mathrm{R}$ & Resistor (linear) & $\mathrm{X}$ & \\
$\mathrm{C}$ & Capacitor (linear) & $\mathrm{X}$ & \\
$\mathrm{L}$ & Inductor (linear) & $\mathrm{X}$ & \\
VCCS & Voltage controlled voltage source & $\mathrm{X}$ & \\
CCCS & Current controlled current source & $\mathrm{X}$ & \\
VCVS & Voltage controlled voltage source & $\mathrm{X}$ & \\
CCVS & Current controlled voltage source & $\mathrm{X}$ & \\
EDD & Equation-Defined Device & $\mathrm{X}$ & \\
$\mathrm{B}$ & B type current source & & $\mathrm{X}$ \\
EQN & Equation block & $\mathrm{X}$ & \\
INOISE & Current noise source & $\mathrm{X}$ & \\
PIN & Subcircuit pin & $\mathrm{X}$ & \\
SUB & Subcircuit & $\mathrm{X}$ & \\
SPARAM & Subcircuit parameter & $\mathrm{X}$ & \\
\hline
\end{tabular}

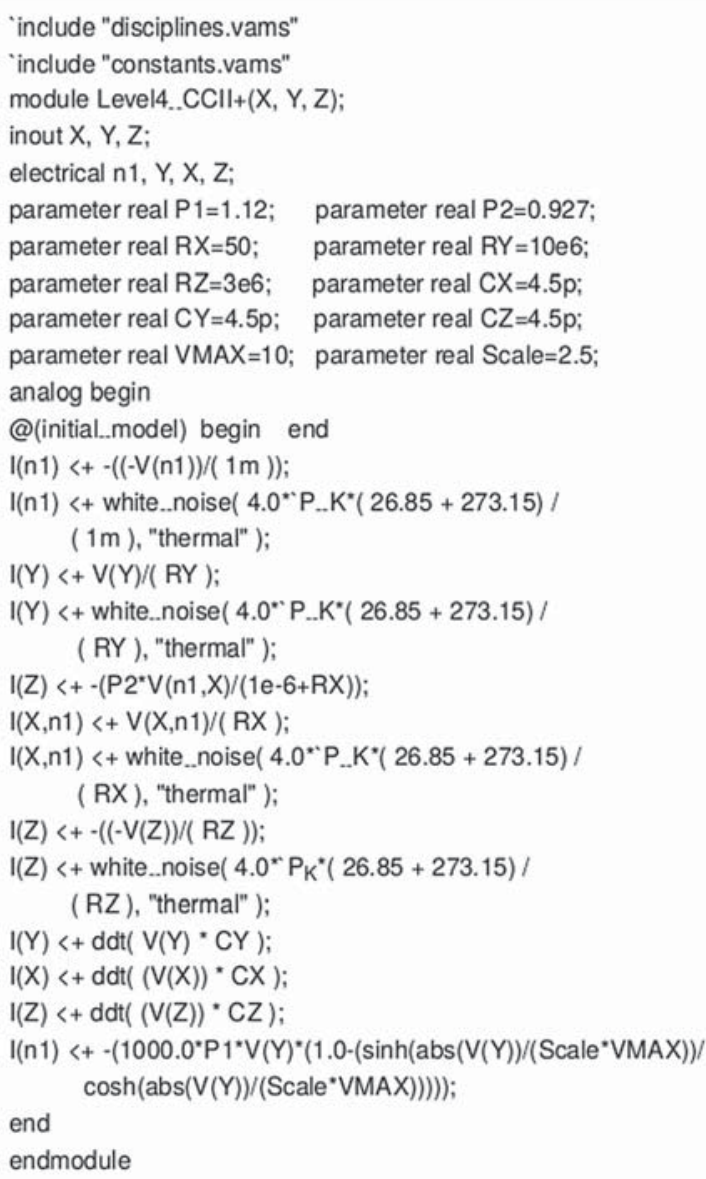

Fig. 8. Qucs Verilog-A module code for a level 5 macromodel synthesized from the level $4 C C I I+$ macromodel given in Fig. 5: for clarity long VerilogA statements have been spread over two consecutive lines.

\section{A COMPARISON OF CURRENT CONVEYOR} BEHAVIOURAL AND SYNTHESIZED CCII+ MACROMODELS IN THE LARGE Signal AC Domain

Illustrated in Fig. 9 are two wideband CCII+ instrumentation amplifiers connected to simulate differential and common mode output voltage signals generated from a resistive signal source. The instrumentation amplifiers are constructed using six level four CCII+ current conveyor macromodes of the type shown in Fig. 5. The instrumentation amplifier gain is given by $R 1 / R 2$ and is set to unity in Fig. 9. The differential gain and common mode gain graphs drawn in Fig. 9 are typical for an instrumentation amplifier where the common mode rejection ratio (CMRR) has a high $\mathrm{dB}$ value at low frequencies then reduces at $20 \mathrm{~dB}$ per decade till a corner frequency is reached where a pole in the CMRR frequency response causes the low frequency zero in the common mode gain to be canceled out.
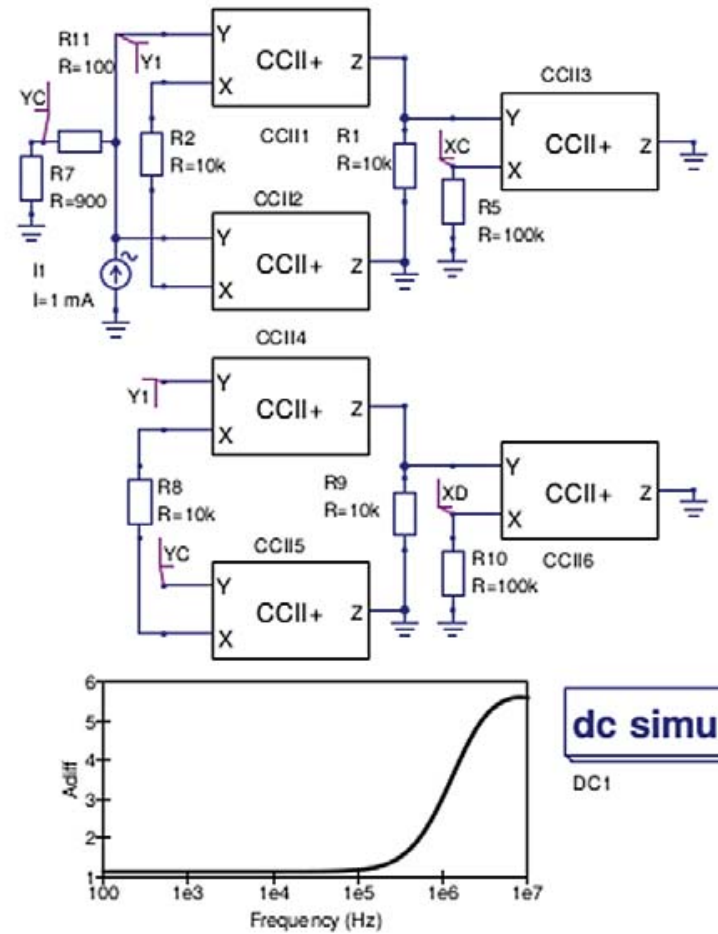

dc simulation

DC

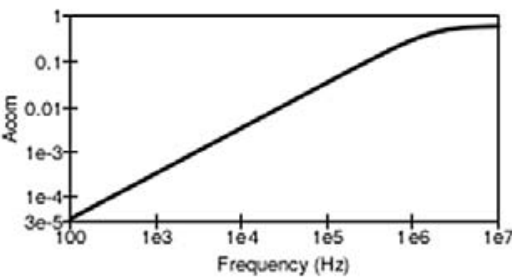

ac simulation $A C$ Type=log Start $=100 \mathrm{~Hz}$ $\mathrm{Stop}=10 \mathrm{~s} \mathrm{HHz}$ Siop $=109 \mathrm{AHz}$
Poin $1 \mathrm{~S}=50001$

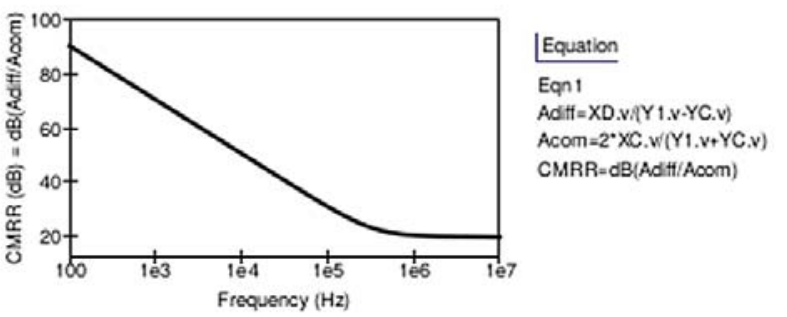

Fig. 9. High bandwidth instrumentation amplifier: Differential and common mode test circuits and typical CMMR simulation data. 
A series of simple timing tests suggest the relative performance figures for the instrumentation amplifier circuit are Qucs Verilog-A = 1, Qucs-S/Ngspice $=3$ and Qucs qucsator $=8$, which suggest that a significant improvement of around eight times, in simulation time, can be gained by synthesizing level five Verilog-A macromodels from level four, or simpler, macromodels.

\section{A STUdy OF CCII+ CURRENT CONVEYOR MaCromodel Large Signal Distortion}

SPICE $3 \mathrm{f} 5$ and a majority of the popular GPL versions of SPICE do not include specific RF and microwave simulation capabilities. Newer SPICE packages based on GPL simulators have often been extended for high frequency circuit and system simulation through the addition of single and multiinput signal Harmonic Balance (HB) analysis of linear and non-linear circuits. The Qucs-S package makes use of the Harmonic Balance simulation features distributed with the Xyce circuit simulator. These HB routines are amongst the most stable available in any of the currently published GPL packages. When coupled with the Xyce .STEP extension it becomes possible to vary input current signals over a range equivalent to small signals to large signals. In Fig. 10 this is done by changing resistor $R 3$ from roughly $100 \mathrm{~K} \Omega$ down to $10 \mathrm{k} \Omega$ (roughly $4 \mathrm{~mA}$ to $40 \mathrm{~mA}$ ). Comparing the output current simulation data illustrated in Fig. 10 with the equivalent values derived from the non-linear current transfer characteristic shown in Fig. 6 suggests that their is good correlation between the output data in the large signal non-linear DC and AC domains.

\section{CONCLUSiOnS}

By combining conventional component simulation models with Qucs Equation-Defined Devices and SPICE B type current sources to form a macromodel, at a stage prior to conversion to a Verilog-A module, it is possible to define a series of five distinct macromodelling levels, increasing modelling flexibility and opening new possibilities in integrated circuit macromodelling. In this paper the concept of model level has been employed to demonstrate integrated circuit macromodel development from simple linear models to complex non-linear models. The conventional macromodel level concept has been extended to introduce an additional model level which adds compact Verilog-A modules at the highest level of the modelling hierarchy. This fifth model level is intended for use with circuit simulators that have VerilogA compiler capabilities for generating $\mathrm{C}++$ model code from Verilog-A modules, generated, by hand or automatic synthesis, from model schematics or indeed, netlists. To demonstrate the use of macromodels the paper introduces, and discusses, the capabilities of a set of five different macromodel cells suitable for simulating RF wideband current conveyor integrated circuits in all simulation domains. A series of CCII+ macromodel circuit test benches are outlined and their relative performance described. The results from these test cases indicate that the proposed macromodel level five Verilog-A module reduces circuit simulation times by a measurable amount which could be significant in the simulation of large non-linear RF current mode integrated circuits.

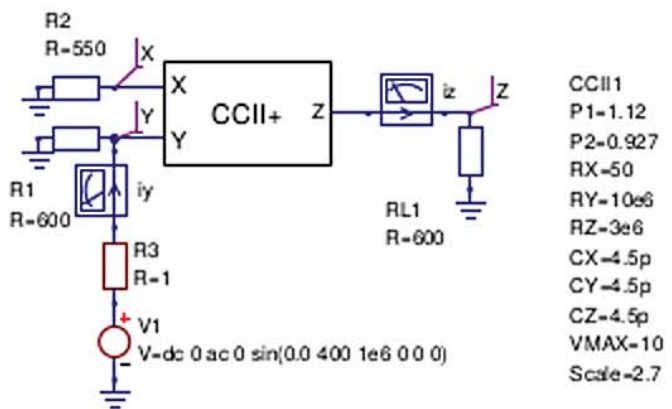

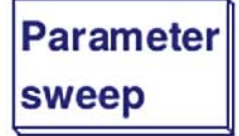

SW1

Sim-HB1

Type-lis:

Param-RO

Values-[99. 4e3; 79.4e3; 59.4e3;39.4e3;19. $4 \mathrm{e} 3,9.94 \mathrm{e} 3]$

SweepMode-tsise
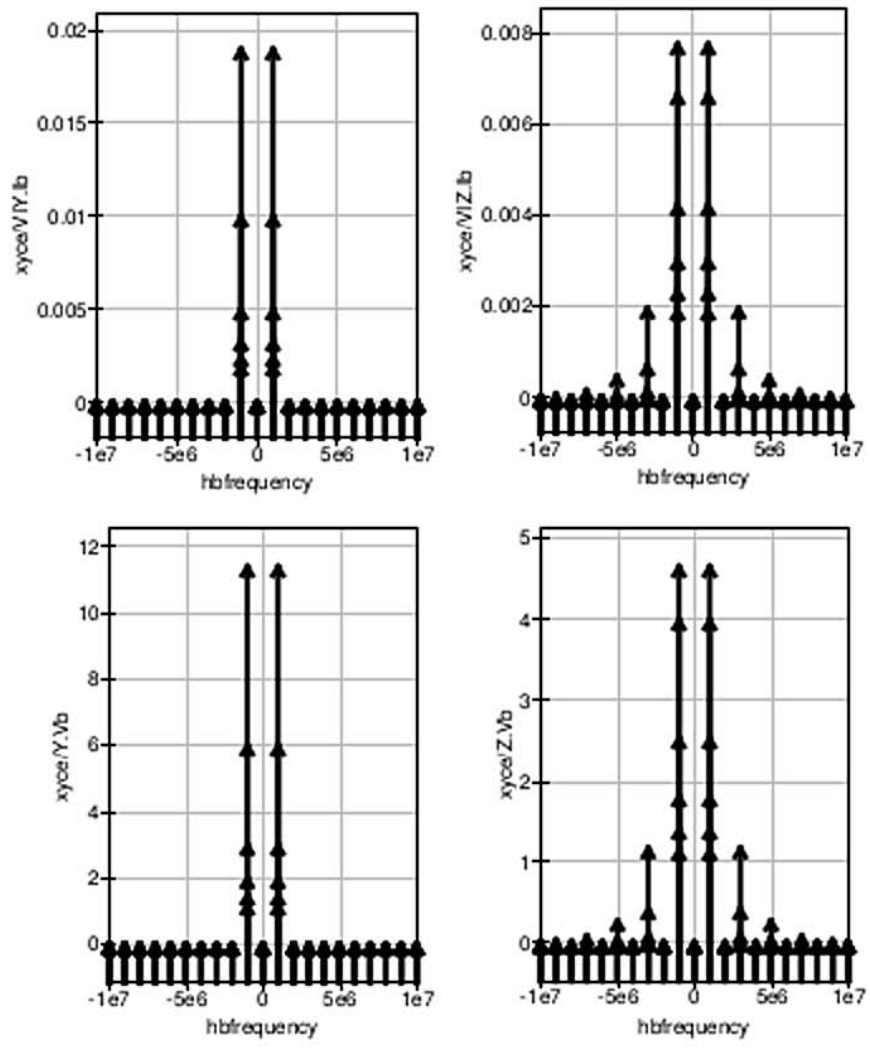

Fig. 10. Test bench for Harmonic Balance simulation of a unity gain level four CCII+ current amplifier and a set of typical simulation data for a single large signal $1 \mathrm{MHz}$ sinusoidal input sign swept over the range $4 \mathrm{~mA}$ peak up to roughly $40 \mathrm{~mA}$ peak.

\section{REFERENCES}

[1] J. A. Connelly and Pyung Choi, "Macromodeling with SPICE", Prentice Hall, Englewood Clifs, New Jersey 07632, 1992.

[2] G.R. Bole, B.M. Cohn, D.O. Pederson and J.E. Soloman, "Macromodelling of integrated circuit operational amplifiers", IEEE Journal of Solid-State Circuits, 1974, vol. 9, pp. 352-363.

[3] S. Jahn and M.E. Brinson, "Interactive Compact Modeling Using Qucs Equation-Defined Devices", Int. J. Numer. Model. 2008, vol 21, pp. 335-349. 
[4] M.E. Brinson and S. Jahn, "Qucs: A GPL software package for circuit simulation, compact device modelling and circuit macromodelling from DC to RF and beyond", Int. J. Numer. Model. 2009, vol 22, pp. 297-319.

[5] Accellera, "Verilog-AMS Language Reference Manual. Version 2.3.1.“, 2009, Available from http://www.accelera.org, [accessed February 2016].

[6] M.E. Brinson, R. Crozier, V. Kuznetsov, C. Novak, B. Roucaries, F. Schreuder. G.T. Torri, "Qucs (Quite universal circuit simulator), Available from http://qucs.sourceforge.net/index.html, [accessed February 2016].

[7] A. Sedra and K. C. Smith, "The Current Conveyor: A new circuit building block", , Aug. 1968, Proc. IEEE, Vol. 576, pp. 1368-1369.

[8] K.C. Smith and A. Sedra, "A second generation current conveyor and its applications", Feb. 1970, IEEE Trans. Circuit Theory, Vol. CT-17, pp. 132-134.

[9] A. Fabre, "Low power current mode second-order bandpass IF filter", June 1997, IEEE Trans. Circuits Syst. II, Vol. 44, pp. 436-445.

[10] B. Wilson, F. J. Lidgey and C, Toumazou, "Current mode signal processing circuits", June 1989, IEEE International Symposium on Circuits abd Systems Proceedings, pp. 2665-2668.

[11] L. Lemaitre, W. Grabinski and C. McAndrew, "Compact device modeling using Verilog-A and ADMS", Electron Technology Internet Journal, Vol. 35, pp. 1-5, 2003.

[12] L. Lemaitre, G. J. Coram, C. McAndrew and K. Kundert, "Extensions to Verilog-A to support compact device modeling", IEEE International Behavioral Modeling and Simulation Conference, BMAS-03, pp. 134138. October 2003.

[13] V. Kuznetsov, "QEP: Qucs schematic simulation with ngspice", 2015, https://github.com/Qucs/qucs/wiki/QEP, (also at: https://github.com/ra3xdh/qucs/tree/spice4qucs), [accessed February 2016].

[14] Brinson M, Crozier R, Kuznetsov V, Novak C, Roucaries B, Schreuder F, and Torri GT. "Qucs: An introduction to the new simulation and compact device modelling features implemented in release 0.0.19/0.0.19Src2 of the popular GPL circuit simulator". MOSAK ESSDERC/ESSCIRC Workshop, 18 September Graz, 2015. http://www.mos-ak.org/graz_2015/presentations/T_5_Brinson_MOSAK_Graz_2015.pdf, [accessed February 2016].

[15] C. Toumazou, F.J. Lidgy and D.G. Haigh (Editors), "Analogue IC design: the current-mode approach", Peter Peregrinus Ltd. on behalf of the Institution of Electrical Engineers, London, UK, 1998.

[16] L. Lemaitre and B. Gu, "ADMS- a fully customizable Verilog-AMS compiler approach", NSTI-Nanotech 2008, www.nsti.org, ISBN 978-14200-8505-1 Vol. 3, pp. 787-790.

[17] V. Kuznetsov and M. E. Brinson, "Qucs-S: Qucs with SPICE", https://ra3xdh.github.io/, [accessed October 2017].

[18] P. Nenzi and H. Vogt. "Ngspice-27 (Next generation SPICE version 27), 2017. [Online] Available: http://ngspice.sourceforge.net/.

[19] Sandia National Laboratories US., "Xyce parallel electronic simulator version 6.7”,2017, [Online] Available: https://xyce.sandia.gov/.

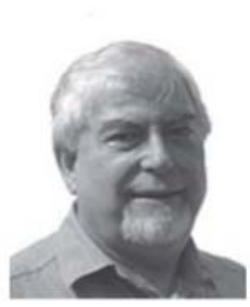

Mike Brinson received a first class honours BSc degree in the Physics and Technology of Electronics from the United Kingdom Council for National Academic Awards in 1965, and a PhD in Solid State Physics from London University in 1968. Since 1968 Dr. Brinson has held academic posts in Electronics and Computer Science. From 1997 till 2000 he was a visiting Professor of Analogue Microelectronics at Hochschule, Breman, Germany. Currently, he is a visiting Professor at the Centre for Communication Technology Research, London Metropolitan University, UK. He is a Chartered Engineer (CEng) and a Fellow of the Institution of Engineering and Technology (FIET), a Chartered Physicist (CPhys), and a member of the Institute of Physics (MInstP). Prof. Brinson Joined the Qucs project development team in 2006, specializing in device and circuit modeling, testing and document preparation.

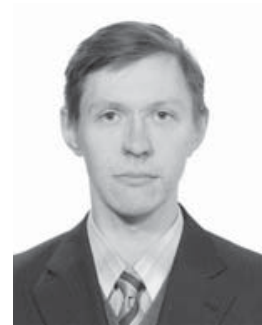

Vadim Kuznetsov was born in Kaluga, Russia in 1988. He received dipl. engineer degree from Moscow Bauman State University (BMSTU) in 2010. He received $\mathrm{PhD}$ degree from Higher school of Economics in 2014. He is Associate Professor of Electronic Engineering department of Kaluga Branch of BMSTU. His research field is electrostatic discharge simulation methods. His field of interest is electronic design automation (EDA) CAD opensource software development. He is core member of Qucs circuit simulator development team. 